\title{
Disimular la feminidad, vestirse de masculinidad. Mujeres operadoras de la industria química de Tarragona
}

\author{
Carla Aguilar-Cunill \\ Universitat Rovira i Virgili \\ carla.aguilar@urv.cat
}

Resumen: El sector industrial sigue siendo un entorno laboral fuertemente masculino, tanto porque los hombres siguen siendo mayoría en número como porque la masculinidad sigue siendo la lógica fundamental en sus procesos productivos. Este artículo analiza la situación actual, en que las trabajadoras, aunque son minoría, bace ya años que participan de este entorno laboral. Concretamente, el análisis está centrado en el nivel de las relaciones informales en el espacio de trabajo para entender el papel que mantiene el género en este entorno laboral. Con este objetivo, se realiza un estudio de caso del polo quimico de Tarragona, el más importante del sur de Europa, en el que actualmente la fuerza de trabajo femenina es el 15\%. El estudio se ciñe especificamente al nivel de operadores/as de planta, los puestos de trabajo de base de la industria, desde los que se controla el proceso quimico in situ. Para la investigación, de base cualitativa, se han becho 14 entrevistas a mujeres que realizan este trabajo, analizadas a través de una perspectiva de género constructivista. La metodología de la investigación en la que se enmarca esta tesis es la investigación-acción, por lo que los resultados aqui presentados también se ban devuelto a las informantes en grupos de discusión para su debate y mejora. El principal resultado de este artículo es que, pese a la creciente participación femenina en el ámbito de la industria química, las dinámicas de relación siguen estando marcadas por la masculinidad. Pese a que la entrada de las mujeres se produjo unos veinte años atrás, se siguen detectando resistencias por parte de los trabajadores a la entrada de las mujeres. Estas resistencias se trasladan al plano identitario de las mujeres, quienes experimentan una cierta contradicción entre ser mujer y ser parte del equipo de operadores. Todo ello hace necesario que las mujeres desarrollen estrategias para el día a día en este entorno laboral, en las que utilizan herramientas de género. Para incorporarse mejor a este entorno laboral, las mujeres desarrollan estrategias coincidentes pese a ser individuales, disimulando elementos propios de la feminidad, a la vez que se visten con elementos masculinos. Así intentan escapar de la bipervi- 
sibilización negativa que tiene la feminidad en este entorno laboral. Mediante esta investigación, se pone sobre la mesa la importancia de las relaciones informales en el trabajo como espacio de dominación. Su gestión diaria, de acuerdo con los resultados, supone una carga extra de trabajo emocional y de estrés que recae sobre las mujeres.

Palabras clave: Masculinidad, industria, igualdad de género, recursos humanos, interacciones informales.

\section{Hide feminity, dress in MASCulinity. Women operators in the CHEMICAL INDUSTRY IN TARRAGONA, SPAIN}

Abstract: The industrial sector continues to be a strongly masculine working environment, both because men continue to be the majority in number and because masculinity is still the fundamental logic in their productive processes. This article analyzes the current situation, in which the women workers, although they are a minority, bave been participating in this work environment for years. Specifically, the analysis focuses on the level of informal relationships in the workplace to understand the role that gender maintains in this work environment. With this objective, this is a case study of the chemical pole of Tarragona, the most important one in southern Europe, where the female work force currently stands at $15 \%$. The study is specifically focused on the level of plant operators, the basic jobs of the industry, from which the chemical process is controlled in situ. For qualitative research, 14 interviews were conducted with women who perform this work analyzed through a constructivist gender perspective. The investigation methodology in which this thesis is framed is the action research, so the results presented here have also been returned to the informants in discussion groups for debate and improvement. The main result of this article is that, despite the growing female participation in the field of the chemical industry, the relationship dynamics are still marked by masculinity. Although the entry of the women took place some twenty years ago, resistance continues to be detected by the workers at the entrance of the women. These resistances are transferred to the identity plane of women, who experience a certain contradiction between being a woman and being part of the team of operators. All this makes it necessary for women to develop day-to-day strategies in this work environment, in which they use gender tools. To better incorporate themselves into this work environment, women develop matching strategies despite being individual, disguising elements of femininity, while "dressing" with masculine elements. This is how they try to escape the negative hypervisibility that femininity has in this work environment. Through this research, the importance of informal relationships at work as a space of domination is put on the table. Its daily management, according to the results, supposes an extra load of emotional work and stress that falls on women.

Keywords: Masculinity, industry, gender equality, buman resources, informal interactions. 


\section{Introducción}

Pese al mantenimiento de la segregación laboral vertical, cada vez cuesta más encontrar entornos laborales únicamente masculinos. Las políticas activas de Gobiernos y empresas se han centrado en los últimos años en aumentar la igualdad mediante la incorporación de las mujeres a espacios laborales tradicionalmente reservados para los hombres. La consideración de la diversidad como un elemento positivo para las empresas (igualmente en cuanto al género) ha promovido la búsqueda de perfiles menos tradicionales también en el ámbito industrial. Pero de acuerdo con Pontón (2014), ello no asegura la disminución de las discriminaciones de género. La forma en la que se haga esta incorporación marcará también las situaciones futuras, así como el bienestar de las personas trabajadoras durante este proceso. Por ello, este artículo analiza este momento de transición, considerado clave, en el que se tiende activamente hacia el objetivo de una mayor presencia femenina, pero esta todavía es muy pequeña.

Para ello, se realizará el estudio de caso de la industria química de Tarragona, centrado, concretamente, en los puestos de trabajo de operadores/as de campo. Es un entorno laboral interesante, ya que pasaron más de veinte años hasta que hubo una mujer trabajando en estas categorías laborales, y actualmente, cuando ya hace aproximadamente otros veinte años que empezaron a ser contratadas, siguen siendo una pequeña minoría en la mayoría de las empresas (según el trabajo de campo, en torno a un $18 \%$ de media). Así, se puede observar que hubo una barrera formal a su entrada (la no contratación de ninguna mujer) que se ha eliminado, pese a que aún no se ha llegado a la igualdad en cuanto a la participación de mujeres y hombres. El hecho de que ahora sí se contraten mujeres no comporta necesariamente la eliminación de todas aquellas creencias basadas en la diferencia entre los sexos y que legitimaron esa ausencia femenina. Por ello, este artículo indagará en torno a las discriminaciones informales e invisibles que persisten $y$ que ya no son ejercidas por la dirección de las empresas, sino que son reproducidas en las dinámicas de relación entre compañeros y compañeras de trabajo. La informalidad de estas cuestiones no debe confundirse con la poca importancia, ya que, de acuerdo con diversas autoras (Acker, 2006; Wright, 2016, Miller, 2004), las relaciones informales en el ámbito laboral constituyen un elemento clave en la reproducción de las desigualdades de clase, género y etnia. Además, y como se desarrollará a lo largo del artículo, también son una fuente de malestar en el trabajo para las mujeres que lo experimentan. Mediante este análisis se plantea que para garantizar una igualdad real es necesario trabajar también sobre este nivel de las relaciones laborales. 


\section{Metodología}

En este artículo se utiliza una metodología basada en el análisis cualitativo de 14 entrevistas llevadas a cabo entre febrero y octubre de 2017 de forma individual y con una duración de entre una y dos horas. Estas constan de una primera parte de cuestionario orientado a la contextualización de las entrevistas y una segunda parte de entrevista semiestructurada. El cuestionario incorporaba preguntas tales como los años que llevaban trabajando en la química, si tenían hijos/as o el número de mujeres con que coincidían en su entorno laboral. En la segunda parte se recorrían los siguientes apartados: su experiencia al incorporarse en la empresa, la evolución de su relación con los compañeros, las diferencias observadas entre hombres y mujeres, la facilidad en la conciliación, las implicaciones de la turnicidad y los elementos de riesgo de su entorno laboral. Sobre las entrevistas se ha desarrollado un análisis del discurso desde una perspectiva feminista constructivista.

Esta investigación forma parte de una investigación más amplia de tesis, y utiliza la primera fase del trabajo de campo: las entrevistas a mujeres operadoras. En las siguientes fases se entrevistará a hombres operadores y a personas directivas. El conjunto del análisis se desarrollará mediante una metodología de diseño propio de tipo investigación-acción participativa, con el objetivo de proponer unas herramientas de mejora para estos entornos laborales. Por ello, los resultados aquí desarrollados no solamente son una producción teórica, sino que también serán devueltos y discutidos con las personas informantes, reconociéndolas como sujetos activos de la investigación, con capacidad de agencia para utilizar sobre su realidad las herramientas provenientes del análisis teórico. En aportaciones posteriores se desarrollarán los resultados de estas discusiones y la potencialidad de la investigación-acción en la mejora de las realidades sociales observadas.

\section{Contexto y presentación de las informantes}

La industria química en Tarragona es el polo químico más importante del Estado y de todo el sur de Europa, produciendo el 50\% (Viu la Química, 2, 2016) de la producción química de todo el Estado español. Para la provincia de Tarragona, es un sector económico fundamental, que comporta el $56 \%$ de las exportaciones desde la provincia (Viu la Química, 2, 2016). Este importante peso económico se traduce en este caso en un importante nicho laboral para la provincia, que mueve 10.000 puestos de trabajo directos y 35.000 indirectos solamente en Tarragona, y 790.000 entre directos e indirectos en toda España (Diari de Tarragona, 13 de abril de 2017). Además, estos puestos no solo son numerosos, sino que son de 
excepcional calidad, especialmente en estos años posteriores a la crisis. Y es que el 95\% de la contratación es indefinida, con un salario medio de 37.550€ (Diari de Tarragona, 13 de abril de 2017). Este nivel salarial está muy por encima del nivel medio del sector industrial, que se sitúa en 27.034€ en España. Además, es un sector con una importante inversión en formación, destinándose de media $253 €$ por trabajador por año (Viu la Química, 2, 2016). Pese a ello, parece que no es un nicho de empleo que reparta sus beneficios a toda la población, ya que, aunque la participación femenina en el sector va aumentando lentamente, actualmente solo supone el $15 \%$ de la fuerza de trabajo. Este bajo porcentaje refleja bien la situación general de la industria en cuanto a su composición de género. Según datos del INE, un 71,25\% de los trabajadores de la industria manufacturera y un $100 \%$ de la extractiva son hombres, y tanto la OIT (2016) como el Banco Mundial (2017) señalan el mantenimiento de la masculinización de este sector laboral, e incluso el crecimiento de su brecha de género.

En este contexto, se han realizado 14 entrevistas a mujeres operadoras de planta y panelistas, que en algunas empresas son puestos diferenciados y en otras, no. Las tareas de las operadoras son controlar los procesos químicos in situ, así como intervenir en dichos procesos cuando es necesario, principalmente abriendo o cerrando, normalmente a mano, las válvulas de las enormes cañerías. Las panelistas realizan también ese control del proceso, pero desde ordenadores ubicados en el lugar de la producción. La producción química es un proceso ininterrumpido durante las 24 horas del día, por lo que estas categorías laborales suponen trabajar en turnos rotativos de mañana, tarde y noche. Este sector productivo, como señalan diferentes informantes de forma muy gráfica, «no es una fábrica de galletas», ya que en él se asumen importantes riesgos, debido a la posibilidad de contacto con productos químicos, inflamables o cancerígenos, así como el riesgo de accidentes graves.

La mayoría de las informantes ha entrado a trabajar a su empresas a partir de las prácticas formativas, canal que parece el habitual para incorporarse en este entorno laboral. Diversas de las entrevistadas han seguido estudiando mientras trabajaban, y en el momento de la entrevista tres de ellas son ingenieras, y una ha acabado también un máster en química. Llevan entre dos y veinte años en la química y trabajan en tres empresas químicas que realizan producción industrial y que forman parte de la Asociación de Empresas Químicas de Tarragona. Doce de las informantes se definen heterosexuales, una de ellas es lesbiana y una, de orientación fluida. Diez de ellas tenían pareja en el momento de la entrevista y cuatro de ellas son madres. Tienen entre 25 y 48 años y viven en la provincia de Tarragona, donde han nacido todas ellas excepto tres, que provienen del 
resto de España. Cuatro de ellas viven solas, siete, en pareja, una lo hace con sus hijas y otra, con sus padres. Entre sus hobbies, la mayoría de ellas destaca deportes, aunque estos son diversos, desde el rugby hasta la natación o la vela, y muchas señalan el gimnasio como su principal forma de desconexión. En general están satisfechas con su trabajo y ninguna de ellas se plantea dejar el sector de la industria química. Doce de ellas son indefinidas y solo dos se encuentran trabajando con contrato temporal, aunque encadenando contratos en la misma empresa. Al ser preguntadas, la mayoría de las informantes se posiciona en contra de las medidas de discriminación positiva en el acceso de las mujeres a este entorno laboral.

\section{Marco teórico: el entorno laboral masculino}

Los procesos de trabajo no solo generan un producto de mercado, sino también una cultura del trabajo propia, que trasciende de ese entorno laboral en el que se desarrolla, afectando e influyendo en los diferentes ámbitos de la vida social e incluso en las mismas concepciones de los individuos sobre sí mismos y los demás (Moreno, 1997). Estas culturas del trabajo «están internamente generizadas y etnizadas» (Moreno, 1997: 22). Por ello, se abordará el espacio de trabajo como un espacio de producción ideológica, en el que sus construcciones sociales específicas determinan fuertemente el acceso de los individuos a los diferentes espacios de trabajo y poder. Trabajar es hacer género en el sentido de West y Zimmerman (1987), ya que ambas acciones son indesligables (Denissen, 2010; Comas, 1995). Porque «las ideas no constituyen simplemente una dimensión subjetiva del trabajo: se trata más bien de estructuras objetivas, que forman parte de la propia substancia y característica del trabajo» (Téllez, 2001:5). Por ello, de acuerdo con Acker (2000), es necesario analizar las estructuras y dinámicas de las organizaciones desde una perspectiva feminista, ya que la misma organización está dotada de género, y este «no es algo que se añade a los procesos actuales concebidos como genéricamente neutros; más bien, es una parte integral de estos procesos que no pueden ser comprendidos de manera adecuada sin un análisis de género» (Acker, 2000: 124). Desde la observación práctica, Palermo (2015) también llega a la misma conclusión, identificando en los discursos analizados un «sujeto-fabril-petrolero-masculino», un individuo abstracto que ya incorpora elementos propios del trabajo industrial y de la masculinidad, al que todas las personas trabajadoras pretenden parecerse. Y es que, además, desde los men studies también se resalta la participación en el ámbito laboral como un elemento fundamental en la construcción de la masculinidad (Connell, 1995), así como en 
ponerla a prueba (Lupton, 2000; Kimmel, 1997), y ello es especialmente visible en los ámbitos de trabajo más masculinizados, como el sector industrial.

Por todo ello, no se partirá de un entorno neutro al género, sino de todo lo contrario, «el supuesto estándar neutral al género es, de hecho, un estándar masculino» (Peterson, 2007: 109). Por ello, el análisis aquí realizado no solo explica las relaciones de género que intervienen en una organización, sino que identifica el género como un elemento fundamental para la comprensión del funcionamiento de este entorno laboral. Además, consideraremos que el entorno laboral analizado es un entorno no solo con mayoría de hombres, sino también masculinizado, de acuerdo con la definición de Collins (2015). Es debido a que no se trata solamente de un entorno en el que numéricamente encontremos una mayoría de hombres sino que, además, normaliza una determinada construcción de la masculinidad. Una forma de masculinidad orientada al riesgo y al trabajo, entendida desde la heterosexualidad. Esta distinción es importante ya que no solamente generará una situación de discriminación sobre las mujeres, sino también sobre los hombres que no encarnen satisfactoriamente los valores del ideal de masculinidad imperante. Es en este sentido en el que la masculinidad puede ser entendida como una competencia laboral, ya que es un elemento que acaba siendo necesario aprender y ejercer para desarrollarse en esos puestos de trabajo. Y es que en el sector industrial encontramos una organización del trabajo que cuenta con elementos propios de la masculinidad (como la fuerza, la resistencia o la dureza física) y de su camaradería específica para desarrollar el trabajo y crear una cohesión en el equipo (Miller, 2004; Palermo, 2017; Collins, 2016). Elementos identificados por Ward (2015) como los deportes de contacto que se realizan en grupo fuera del trabajo, el «cotilleo», los relatos sexuales, beber grandes cantidades de alcohol o la visibilización de la heterosexualidad y la homofobia aparecen recurrentemente en las investigaciones sobre la masculinidad en el trabajo industrial y son la base de ese espacio de complicidad conocido como camaradería masculina.

Ello crea una brecha de poder y una estructura de dominación de lo masculino sobre lo femenino, y los cuerpos de mujer son los que tienen un mayor hándicap de entrada. Pese a ello, esta estructura de poder es legitimada y asumida por toda la estructura laboral, y difícilmente es cuestionada, sino más bien lo contrario, ya que el «consenso entre hombres y mujeres sobre el ideal dominante de masculinidad implica unas relaciones de poder estables entre hombres y mujeres» (Stobbe, 2005: 111). Para observar estos elementos, ya en diversas investigaciones sobre mujeres en entornos masculinizados observamos que las interacciones diarias son un elemento clave en el análisis de la participación de mujeres en entornos masculinizados. Miller (2004) analiza a las mujeres trabajadoras de 
la industria petrolera e identifica que las mujeres experimentan un sistema de valores masculino, que crea un conflicto entre los «roles femeninos»y los «roles normales» (masculinos). Las interacciones diarias son uno de los ámbitos en los cuales las mujeres sienten esa contradicción, ya que no son tratadas como una más, sino que se encuentran con actitudes paternalistas o caballerosas. En el estudio de Wright (2014) en torno a las mujeres del mundo de la construcción y el transporte, observa la enorme importancia de las interacciones diarias en el entorno laboral como una forma de estructurar las experiencias de las mujeres. La autora observa que existen diferentes actitudes en torno a entablar relaciones estrechas entre mujeres, ya que pueden ser entendidas como una falta de lealtad hacia los hombres, y una dificultad en el camino de las trabajadoras para llegar a ser «uno más». De acuerdo con Kanter (1977), esto supondría un refuerzo de su identificación como «chicas» o mujeres, lo cual puede no ser muy deseable en contextos de trabajo masculinizados. En las diferentes investigaciones, pues, observamos una cierta contradicción entre feminidad y masculinidad, y mientras la identidad masculina se solapa con la de la persona trabajadora, la identidad femenina cuesta encajarla. A continuación desarrollaremos esta idea mediante los resultados del trabajo de campo.

\section{5. ¿Se puede ser mujer y operadora?}

La entrada de mujeres a los espacios de trabajo de la industria química supone aún hoy una desestabilización del equilibrio social interno de este espacio de trabajo. Un estudio anterior ya señala la existencia de discursos en este entorno laboral que legitiman que existan trabajos de hombres y de mujeres (Pontón, 2014). Ello cristaliza en las entrevistas como un conflicto interno en las mujeres entre ser mujer y ser operadora o panelista. Parece existir una contradicción entre las categorías «mujer» $y$ «operadora» o, lo que es lo mismo, una imposibilidad conceptual de ser ambas cosas. Se observa en el uso del lenguaje durante la entrevista, ya que en muchos casos espontáneamente las trabajadoras utilizan ser «uno más» en masculino y «ser una mujer» expresa el estar fuera del grupo. También aparece expresada la dificultad que experimentan para ser como un compañero más siendo mujer. Un ejemplo de ello es Esther, al explicar que en sus primeros años en la industria ella reivindicaba lo siguiente: «Soy una compañera más, soy una compañera, no una mujer. Un compañero más de trabajo!» (Esther). De hecho, ser una más es un horizonte que algunas señalan como imposible: «Y entonces ya te miran como una igual. Pero bueno..., como igual como mujer, al final. Al final seguías siendo una mujer. Que el papel [de mujer] estaba clarísimo pero al menos estaba aceptada» (Mari). Ello indica que 
la normalidad sigue correspondiendo a los trabajadores masculinos, y que ellas siguen siendo las excepciones.

$\mathrm{Y}$ es que mientras solo había hombres trabajadores, el género podría considerarse un elemento aparentemente ausente, pero a partir de lo que relatan las informantes, la masculinidad debía vehicular de forma importante las relaciones entre los hombres trabajadores. Las relaciones laborales y los espacios de trabajo seguían lógicas de la masculinidad y la camaradería masculina, probablemente en una situación parecida a la que relata Palermo (2017) para la industria petrolera argentina, en la que actualmente solo encontramos trabajadores hombres. En todo caso, en la actualidad se mantiene esa normalización de la masculinidad en el lugar de trabajo, y un indicador es que todavía se mantiene la idea de que las mujeres no son capaces de realizar el trabajo industrial. Y no es una idea vaga o inconsciente, sino que, a día de hoy, a prácticamente todas las informantes se las ha cuestionado de forma más o menos directa sobre su capacidad para realizar el trabajo para el que han sido contratadas por el hecho de ser mujeres: «Un compañero que tenía yo me decía en la cara que no veía bien que hubiera mujeres en la refinería de operadoras. Que no podíamos hacer bien ese trabajo» (Casandra).

En la práctica, la suposición de que las mujeres no son capaces de hacer determinadas tareas se traduce en dos actitudes principales: por un lado, el paternalismo, y por el otro, la puesta a prueba, que se dan especialmente los primeros años de trabajo en la industria química. En el primer caso, los compañeros no dejan que las mujeres realicen ningún trabajo solas, dando a entender de forma muy sutil que las consideran incapaces de hacerlo o que es demasiado peligroso o pesado para ellas. En algunos casos se hace de forma muy ofensiva y en otras, de forma cortés o protectora. En cualquier caso, se presta una ayuda que ellas no han solicitado, $y$ antes incluso de que tengan tiempo de empezar a realizar la tarea. «Por una parte era la princesa o la niña $[. .$.$] , pero por$ otra parte, mucho machismo. Mucho. De querer abrir una válvula y "aparta, que tú no puedes". De querer hacer algo y..., "ya lo hago yo"» (África). Las respuestas de las mujeres van desde la resistencia y la demostración de su capacidad hasta la aceptación, o también la naturalización de su menor fuerza debido a un cuerpo más pequeño o a una mayor fuerza de sus compañeros en concreto o de los hombres en general. En ocasiones se lo toman como una ofensa y en otras, como una ayuda o una galantería, incluso como un cuidado. Por lo que, pese a que la actitud por parte de los hombres sí parece generalizada, la respuesta femenina muestra más matices. En el segundo caso, cuando la suposición de que ellas no pueden realizar determinados trabajos supone ponerlas a prueba, 
los compañeros hacen lo contrario: dejan las tareas más duras a las mujeres, y en ocasiones contemplan ostensiblemente cómo ellas solas realizan una tarea muy pesada. En esas ocasiones puede aparecer en los relatos la intervención de superiores jerárquicos, que obligan a los compañeros a prestar ayuda a la mujer, ya que las situaciones pueden convertirse en peligrosas. En estas ocasiones, las respuestas de las mujeres observadas son más homogéneas, aceptan el reto y responden trabajando mucho más duro y evitando pedir ayuda. Generalmente las explicaciones de por qué lo hacen se sitúan en la propia personalidad terca o en una demostración propia de capacidad. Incluso en algunos relatos aparece la constatación directa de que ellas tienen que trabajar mucho más que los hombres para ganarse su respeto. "Tenemos que trabajar más para que se den cuenta de que podemos trabajar igual» (Mari). Estas puestas a prueba suponen un agotamiento físico y emocional extra en el caso de las mujeres, y se dan muy especialmente en los primeros años del trabajo en un equipo de operadores. Una vez han probado su valía, este tipo de situaciones parecen espaciarse e incluso desaparecer.

La heterosexualidad es uno de los elementos que sustentan las relaciones de camaradería masculina. La muestra de esta heterosexualidad es evidenciar interés sexual por las mujeres del entorno, que las mujeres lo relatan como cuestiones más o menos sutiles, que las mujeres normalizan y trampean como pueden cuando no les interesa. "Así tontear, sí. Ya no te digo nada si te giras y miras si te están mirando o no porque no me dedico a ello, pero sí que es así. Que, a ver, que no le das más importancia porque creo que nos hemos acostumbrado. Nos hemos acostumbrado» (María). Pero más allá del tonteo o el ligue, son más preocupantes las situaciones en las que los trabajadores hablan de forma muy sexual sobre las mujeres, lo cual supone una incomodidad e incluso una cierta amenaza a la mujer que escucha, ya que, en ocasiones, ellas mismas pasan a ser el blanco de esas conductas. En el trabajo de campo se han recogido algunas situaciones fácilmente susceptibles de considerarse acoso sexual de tipo verbal, de acuerdo con el marco legal aplicable recogido en el Protocolo sobre acoso sexual y por razón de sexo en la empresa elaborado por la Generalitat de Catalunya (2015). Las situaciones narradas, aunque en muchos casos se explican con desenfado, llegan a ser muy violentas, y debemos tener en cuenta que se dan en un entorno hostil, ya que trabajan en lugares aislados y en los que por las noches o fines de semana hay pocas personas en kilómetros a la redonda, y las que hay son hombres. Danae relata muy bien esa peligrosidad: «En los turnos de noche yo estaba sola y a lo mejor tenía trabajando a cincuenta tíos en mi planta. Cincuenta tíos y he tenido que sufrir el ir paseando por allí por planta y tener 
que coger una llave de válvula de esas de cinco quilos porque la gente me silbaba o los tíos me decían: "Guapa, cuando puedas, pásate por aquí...”. A ver, si tú estás viviendo eso, tú dices: "O me comes o te como". No hay más. Y yo dije: "Hasta aquí hemos llegado. Si te tengo que pegar con la llave te pego con la llave. Y si te echo de planta, te echo de planta"». Como defienden Wilson et alii (2001), estas situaciones son el ejercicio de una dominación masculina que, según las entrevistas, en muchos casos contaba con la connivencia y participación del resto de compañeros, aumentando la indefensión de la trabajadora. La repetición de los comentarios en diversos días aumenta la tensión de la situación y abre la posibilidad de que trascienda del plano verbal. A continuación seguimos uno de los casos relatados:

Entonces hubo un día que me dijeron: «¿Qué tal si nos vamos a la vaca [un espacio de descanso] y te zumbo?». «¿Perdona?». «Sí, sí, pim pam, pim pam, echamos un polvo y ya está». Y te quedas así como diciendoः « $Y$ ahora qué te

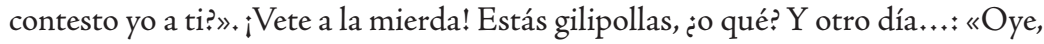
¿qué te parece si hacemos un bucaque?»+ ¿Se llama? Aquello que se pone la mujer en medio y todos se le corren en la cara. Y te quedas: «¿De verdad me estás soltando estas burradas así de buenas a primeras?». Y claro, uno lo suelta, el otro lo potencia, y a ver quién la suelta más basta (Dora).

La informante, al no admitirles esta situación de violencia verbal, pareció generar ella el conflicto, cortó la diversión, y sus compañeros criticaron su incapacidad para aceptar una broma. Otras mujeres explican cómo han hecho un proceso consciente de adaptación a este tipo de situaciones, generando una capacidad de respuesta en la misma línea: "Hoy se te oye la voz que parece que no te la han metido". Me sobra. Me sobra, no me hace falta esto. Pero bueno, yo era más burra y decía: "Sí, me la han metido hasta donde me la tenían que meter". Es que vi que o me ponía al mismo nivel o me iban a comer» (Mari). En todo caso, son situaciones tensas y violentas para ellas, que en muchos casos parecen darse con cierta normalidad y de forma generalizada. Pese a ello, y precisamente por la normalización de estas cuestiones, muy pocas de ellas han informado a sus superiores jerárquicos, ni conocen casos cercanos que lo hayan hecho. De hecho, pocas de ellas identifican estas situaciones como de acoso sexual.

En contraposición a estas situaciones, hay una informante que relata su incorporación al equipo a través de una lógica de parentesco. Define las relaciones con sus compañeros como las propias de una hermana menor, y dice sentirse muy cómoda con ello. Esta lógica familiar parece crear un entorno cómodo y seguro para la informante, y la protege de los comentarios sexuales. Pero si, como hemos visto, la sexualización constituye una forma de dominación, esta forma 
de relación, aunque elimina el factor sexual e incorpora una importante carga afectiva, no elimina el factor de ascendente de los hombres sobre la mujer, ya que, si ella es una hermana menor, ellos son hermanos mayores. De esta forma, vemos cómo las relaciones que se establecen son diversas, pero mantienen un elemento de desigualdad, en el que los hombres juegan un papel dominante o paternalista mientras la mujer es objetualizada o infantilizada. Ello denota la dificultad de desarrollar un compañerismo laboral que pueda incorporar a las mujeres con naturalidad y en igualdad, lo cual debería partir de un reconocimiento de las capacidades también de las mujeres para desarrollar el trabajo de operadora de planta.

Otro elemento a tener en cuenta, y que aparece de forma recurrente en las entrevistas, es la rumorología. A las informantes les llama la atención el hecho de que los hombres sean cotillas y comenten mucho sobre el resto del personal, y lo remarcan como una curiosidad. Desde la investigación, llama la atención que las mujeres son un objetivo bastante habitual en estos comentarios. Una de las informantes ha sido continuamente el blanco de rumores falsos sobre sus relaciones con compañeros y otra de ellas mantiene su relación amorosa en secreto por evitar este tipo de problemas. Adela supo años después de entrar en la empresa que sus compañeros pensaban que era lesbiana y este tema había sido comentado durante tiempo antes de que se lo preguntaran y les dijera que es heterosexual. Y Dora explica su experiencia: «Estaba trabajando y cuando llegué me encontré que estaba merendando mi compañera, y, tal y como entré, me dijo: “'Sabes lo que me acaba de preguntar él? Que cómo tienes las tetas, que cómo son tus pezones y si te depilas"» (Dora). En su caso, esta situación comportó un enfrentamiento directo con el hombre al que se hacía alusión y un conflicto entre ellos que duró mucho tiempo. Sus experiencias en este terreno han llevado a varias de ellas a tener un posicionamiento firme ante estas situaciones. Adela remarca su actitud activa de no dar crédito a los rumores que los compañeros muchas veces le explican, ya que considera que es la forma de no dar pie a que se extiendan lo que muchas veces son mentiras, como pasó en su caso. Gemma, en cambio, ha desarrollado la respuesta contraria: mantenerse presente en este tipo de conversaciones, lo que le permite entrar en una relación de camaradería en la que, aunque solo sea escuchando, forma parte del secreto compartido, y participa de esas redes de complicidad. Pero ella misma reconoce en ello un arma de doble filo, ya que, al escuchar según qué comentarios, ve también la amenaza de que en algún momento se giren contra ella: «Ves comentarios que tienen entre ellos o comentarios que tienen con otras compañeras y dices: "Quizá esto también va por mí, ¿no? Y cuando me voy quizá me sueltan una chinada” (Gemma). 


\section{El uso estratégico del género}

Ante unas situaciones en las que ser mujer y trabajadora industrial parecen elementos incompatibles, las mujeres despliegan diversas estrategias para incorporarse al grupo de trabajadores, en las que no solo se trata de demostrar su capacidad laboral, sino también su capacidad de moverse en unas estructuras de relación masculinas. Estas estrategias son fundamentalmente individuales, en contraste con otros procesos de integración de mujeres en espacios masculinos, como las estibadoras recogidas por Aguado (2016), que han utilizado estrategias más colectivas. Concretamente, las mujeres entrevistadas controlan y gestionan los elementos que puedan ser susceptibles de generización (masculina o femenina) tanto estéticos como actitudinales. Cuanto más se acerquen a la normalidad masculina, se pongan ese traje simbólico, más desapercibidas pasarán y se evitarán situaciones desagradables que podrían hacer su día a día más duro. En este proceso, las trabajadoras realizan un vasto trabajo emocional en el que aprenden a normalizar un entorno que les es hostil, a partir de la anticipación de las reacciones de los hombres y el desarrollo de mejores respuestas ante las situaciones violentas. Pero la cuestión es más compleja, y mientras ellas mismas valoran elementos propios de la feminidad como importantes para realizar bien su trabajo, también requieren de elementos propios de la masculinidad para encajar en sus equipos de trabajo.

\subsection{Disimular la feminidad}

La normalización de la masculinidad provoca una exagerada visibilización de la feminidad, ya que es la excepción. Y una excepción que, como hemos visto, parece incompatible con el compañerismo obrero. Esto provoca que las mujeres busquen diferentes formas de encajar lo que en muchas ocasiones parecen vivir como un doble rol: ser trabajadoras y mujeres. $Y$ es que es importante señalar que, como podemos comprobar, el hecho de que las mujeres se incorporen a los entornos laborales tradicionalmente masculinos de la industria química no supone, de forma inmediata, la incorporación de la feminidad a estos entornos, sino que esta sigue siendo rechazada y ellas se incorporan a unas dinámicas de relación masculinas. En ese sentido, es lógico que las estrategias que las mujeres desarrollan para encajar con sus compañeros en muchos casos pasen por esconder o disimular elementos femeninos que en este entorno laboral serían muy llamativos, en todo un proceso observado de gestión de su feminidad en este entorno laboral.

La visibilización de la feminidad que acompaña la entrada de las mujeres a este mundo laboral genera reflexiones y negociaciones personales en torno a ele- 
mentos estéticos vinculados al género, como la forma de vestir, la forma de hablar o el ir o no maquillada. Todas las informantes conocen perfectamente el fuerte control que ejercen sus compañeros (y, en algunos casos, compañeras) sobre estos elementos. Un control que en muchos casos se evidencia con interpelaciones directas, como la situación que le ocurrió a Mari a raíz de una cena de empresa: «Y se comentaba durante toda la semana. No era solo ese día que te hacían la ola. Hala, hala, hala [... . ¿ ¿Por qué? ¿Porque lleve escote? Sí, llevo escote..., mira, una vez y ya está, pero no estés todo el rato: "Hala qué tetas, hala, qué tetorras"». A partir de estas situaciones, las mujeres mantienen o eliminan determinados elementos de una forma consciente, buscando reivindicar su libertad en algunos casos o evitar situaciones incómodas en otros, que, como vemos, pueden ocurrir tanto por llevarlos como por no llevarlos. A todo esto, debe ser tenido en cuenta que son personas que trabajan (salvo excepciones temporales) vestidas de uniforme, por lo que los elementos que pueden modificar son muy limitados. Se reducen prácticamente al maquillaje, el peinado, las uñas y el hecho de llevar más o menos abierta la camisa del uniforme. Es muy interesante cómo estas cuestiones son explicadas en relación a la predisposición al trabajo que sugieren. Así, por ejemplo, el uso de coleta puede mostrar más predisposición al trabajo físico que ir bien peinada, o unas uñas pintadas pueden ser criticadas por no mostrar una capacidad de ensuciarse las manos. Como en la siguiente cita de Gemma: «Por ejemplo, las uñas, a mí no me gusta pintármelas porque es como que haces algo y no sé. Queda repipi». Ese repipi es una feminidad caricaturizada, que, como ella expresa, le crea rechazo. Estas explicaciones muestran cómo las operadoras han integrado la misma lógica masculina que las había rechazado como mujeres, aplicándola a elementos concretos de su feminidad y evitándolos.

En este sentido, entre las informantes entrevistadas, encontramos dos excepciones, Aina y Ángeles. Ellas explicitan una voluntad de mantener y reivindicar una forma normativa de ser mujer, vinculada a unas formas de hacer y de relacionarse, así como a unos elementos estéticos. Quizá no de forma casual son las dos informantes más jóvenes, lo que puede indicar un cambio generacional. En todo caso, son las trabajadoras que más se aproximan a exigir la inclusión no solo de las mujeres, sino de la feminidad expresada de forma tradicional en su entorno laboral y que lo están llevando a cabo de forma activa. Su resistencia es paradoxal para los referentes tradicionales del feminismo, ya que utilizan elementos que a priori parecen lo contrario a reivindicaciones feministas, como llevar las uñas pintadas o ir maquilladas. En su caso, la reificación de la feminidad en un entorno masculino es un discurso de resistencia de una forma de vida propia, identificada como esencialmente femenina, y de la reivindicación 
de su derecho a no tener que modificar sus formas de hacer género por estar trabajando en ese entorno laboral. "Es que tengo que hacerme las uñas $y$ ponerme rímel para al menos llevar algo de que soy una mujer, sí. Que a la gente le hace mucha gracia porque vas siempre en guantes. Y espera, que te firmo un papel. Y me quito un guante y cojo el papel y no sé qué y: "Uy, qué uñas, mírala ella, no sé qué". Y yo: "Hombre, antes muerta que sencilla". Necesito llevar algún toque mío» (Aina). Ellas han mantenido su costumbre de ir maquilladas o peinadas al lugar de trabajo, incorporando incluso una mayor visibilidad de estos elementos. «Yo las uñas nunca me las pintaba antes de trabajar. Ahora sí porque noto que es algo un poquito femenino que puedo tener cuando voy a trabajar» (Aina). Sus dos experiencias son divergentes en cuanto a la forma en que se han recibido esos elementos femeninos. En el caso de Aina sus formas femeninas han sido muy bien valoradas por sus compañeros masculinos, y en la entrevista remarca las felicitaciones que ha recibido por introducir un mayor orden en el lugar de trabajo (que ella identifica como una cualidad femenina) o temas de conversación más íntimos. Pero en el caso de Ángeles, esta resistencia ha suscitado las críticas y la incomprensión de sus compañeros y compañeras: «Yo al trabajo voy supermaquillada, con mi modelito, mi colita, no sé qué..., y te ven y dicen: "¿A dónde vas? ¿A dónde vas? ¿Qué te crees, que vas al mango a vender ropa?"»(Ángeles).

Otro elemento que aparece de forma repetitiva es la gestión de la ropa de calle. Una cuestión que llama la atención, ya que es la ropa que usan únicamente hasta llegar al vestuario, no para usar durante la jornada laboral, pero que siempre se incluye entre los elementos que más tienen en cuenta. Esto denota el grado de control que existe sobre sus cuerpos, que traspasa los muros de la industria y llega hasta horas antes y después del trabajo, ya que dejar de llevar determinadas prendas de vestir, como minifaldas, o no ir maquilladas al trabajo, en ocasiones les supone tener que pasar después por casa para cambiarse cuando tienen una cena o evento nocturno o no poder llevar cierta ropa horas antes del trabajo con la previsión de que tendrán que ir a trabajar directamente. Existe una tensión interna de fondo entre el ejercicio de su libertad individual y la protección ante comentarios sobre su cuerpo que crea contradicciones en el discurso y probablemente en la práctica, como podemos observar en entrevista de Adela, que por un lado afirma: «Si me pilla un día de refinería que voy en vestido y con tacones, voy en vestido y con tacones. [...] Es que me niego. Me niego a tener que depender encima de esto». Pero poco tiempo después matiza: «Sí que es verdad que a veces dices: "Hay que vigilar un poco". Pero por el ambiente de trabajo. $\mathrm{O}$ sea, si yo tuviera que ir vestida de ropa de calle, seguramente no vestiría como 
visto al día a día. Pero porque a mí me han hecho sentir muy incómoda». Ángeles muestra la misma contradicción en su discurso, en el que destaca la libertad de cada persona a vestir como quiera, pero a la vez, que es importante conocer las repercusiones de la ropa. "Yo creo que cada persona se tiene que vestir como quiera en el momento que quiera. Y si los demás miran, los que van a sufrir más son ellos. [...]. Pero yo por ejemplo no me lo pondría porque donde voy sé el círculo que tengo». Como bien expresa Ángeles, cada mujer gestiona cada pieza de ropa o cada estilo en el vestir a su manera, encontrando un lugar propio de equilibrio o comodidad personal, que defienden y explican en el contexto de esta dicotomía entre libertad personal y autoprotección. En muchos casos explican cómo estos equilibrios personales van evolucionando a lo largo del tiempo y pasan temporadas en que visten de una forma más llamativa y otras en que menos.

\subsection{Vestirse de masculinidad}

Los uniformes utilizados por las trabajadoras y trabajadores tienen el mismo corte, un corte que muchas describen como masculino porque no es entallado. Este hecho parece una metáfora de lo que muchas ven necesario hacer en su día a día en el trabajo industrial: vestirse de masculinidad. $\mathrm{Y}$ es que, mientras existe una hipervisibilización de cualquier elemento femenino, también existe un efecto de invisibilización a través de lo masculino. Ello se debe a que, si lo masculino es la normalidad, la mejor forma de llegar a ser uno más y no llamar la atención por ser mujer es integrándose en esa masculinidad. Danae lo reivindica así: «Aquí soy uno más, ya no soy una, soy uno más. $Y$ me tenéis que respetar $y$ me tienes que hablar igual que le hablas a los otros compañeros». Así, en las entrevistas aparecen referencias a la masculinidad que ejercen en este entorno laboral, tanto de forma consciente como inconsciente. En algunas entrevistas incluso son las mujeres las que verbalizan que han observado una masculinización de sí mismas: «Te vuelves más masculina. Mucho» (Casandra); «La verdad es que sí, que me he vuelto más masculina» (Esther).

Mientras los elementos que se gestionan en el caso de la feminidad son elementos estéticos o físicos, visibles, dotados de una materialidad, los elementos que se masculinizan son mucho más sutiles e invisibles, vinculados a la actitud, a la forma de hacer las cosas. En este sentido, resaltan mucho la necesidad de desarrollar una forma más autoritaria de moverse, de hablar, de utilizar el tono de voz. «Mi tono de voz es más autoritario, digamos. No siempre pero sí que tengo que estar muy segura de lo que digo» (Adela). También aparece de forma recurrente la incorporación de una manera de hablar más ordinaria, en la que se 
esquivan las cuestiones sexuales con comentarios aún más vulgares. Consideran estas cuestiones como herramientas necesarias para moverse en ese entorno y, aunque no les gusten, las consideran aliadas para poder ser respetadas por sus compañeros. Es muy evidente su carácter instrumental en la delimitación que hacen de su uso solo en las horas de trabajo, esforzándose por no usarlas en su vida personal. "Quieras que no, entre una cosa y otra se te pegan las costumbres de los compañeros que tienes, y claro..., vas diferente. Y según cuándo, llego a casa y digo: "Esto no lo puedo decir, no lo puedo decir...”. Digo, madre mía, según qué hago y según qué digo, pensarán: “Esta no es mi madre!” (Esther). Aunque este salto no siempre es posible y muchas explican comentarios de personas de su entorno familiar o de amistades que les señalan estos comportamientos más masculinos, aunque es una cuestión que no aparece en las entrevistas como una cuestión que les preocupe, sino más bien que les sorprende porque ellas no se dan cuenta. De esta manera encontramos otra contradicción de género sobre estas mujeres, ya que, mientras en su entorno laboral se señala su feminidad, en su entorno personal se visibiliza su masculinidad. Esto ocurre también en sus entornos de amistades femeninas, lo cual comporta que se salgan un poco del ser unas más entre ellas, al señalarse su masculinización. Por ello, su situación de outsider se mantiene tanto en el grupo de hombres como en los de mujeres, y tanto en su entorno laboral como en su entorno personal.

A la vez, mediante esta masculinización se hacen menos visibles en el entorno de trabajo pero siguen siendo en muchos casos blanco de críticas por parte de sus compañeros. Y es que se da la paradoja de que encuentran dificultades no solo cuando son demasiado femeninas sino también cuando son demasiado masculinas. En algunas informantes esta situación genera impotencia ante las críticas y la imposibilidad de encontrar una actitud en la que no sean criticadas. Casandra explica en la entrevista cómo buscó un tono de voz más autoritario para dar las órdenes y ser respetada, y cómo fue criticada tanto por ser amable como por ser autoritaria: "Vas viendo el tono en el que tienes que dirigirte. $\mathrm{Y}$ pasas de ser la niña que sonríe a la niña que va con la cara de perro y pasas de "niñata" a "malfollada", pero bueno» (Casandra). Danae incluso tiene la opinión de que su masculinización en el trato con el resto de trabajadores (que ella misma explica como necesaria para trabajar donde está) ha sido decisiva para no ser ascendida en un proceso de promoción: «Pues mi jefe consideraba que yo no tenía un carácter lo suficientemente dócil y manipulable, visto desde mi punto de vista, claro, y que pues eso, que yo no tenía suficiente mano izquierda para poder llevar a tanto personal». Sus equilibrios, como vemos, no son fáciles. 


\section{Conclusiones}

Las mujeres que se incorporan al trabajo industrial de la química en Tarragona se incorporan a un entorno laboral en el que la masculinidad sigue estando normalizada. Por ello, se da la paradoja de que se están incorporando las mujeres pero no tanto la feminidad. La camaradería masculina sigue siendo el código que marca las relaciones informales del equipo de trabajo, y las mujeres no son incorporadas de forma natural, sino que realizan un trabajo consciente para incorporarse en estos marcos de relaciones. Esta dinámica parece irse atenuando con los años, posiblemente debido a la creciente presencia de las mujeres en estos espacios laborales, así como a la incorporación de nuevas generaciones de trabajadores que solamente han conocido un entorno laboral mixto. Pese a ello, las experiencias de las informantes indican que siguen existiendo importantes resistencias a la presencia femenina basadas en una devaluación de la mujer en estos espacios laborales. Ante estas situaciones, las mujeres entrevistadas describen un duro proceso de incorporación en el que han utilizado elementos propios de la feminidad y la masculinidad como herramientas para poder encajar en unas dinámicas de relación masculinas. Todo este proceso se lleva a cabo de forma individual por cada una de las mujeres, y es un proceso de incorporación en esas relaciones masculinas, por lo que no las cuestionan, sino que las reproducen y las refuerzan.

La perspectiva tokenista considera que, al ir aumentando el número de mujeres en un entorno laboral tradicionalmente masculino, disminuirán de forma natural las discriminaciones hacia las mujeres en ese entorno laboral (Zimmer, 1988). De acuerdo con los resultados de este estudio, pese a que se ha observado una mejora a lo largo de los años, también se ha detectado que estas mujeres están asumiendo un importante coste emocional en el proceso. Por ello, sería interesante la creación de herramientas de ayuda a esta incorporación, dirigidas a toda la plantilla, para facilitar este proceso. La investigación, pues, continuará en este sentido, procurando un buen diagnóstico mediante las entrevistas a hombres trabajadores y a directivos de diferentes empresas, para, posteriormente, poder desarrollar herramientas construidas en conjunto con las personas que pertenecen a este ámbito, que sirvan para la mejora y promoción del proceso de incorporación de mujeres a la industria química. 


\section{Bibliografía}

Acker, Joan (2006). «Inequality Regimes: Gender, class, and race in organizations». Gender E Society, 20(4): 441-464.

- (2000). «Jerarquías, trabajos y cuerpos: una teoría sobre las organizaciones dotadas de género». En: Navarro y Stimpson (comps.) (2000). Cambios sociales, económicos y culturales. El Salvador: Fondo de cultura económica de Argentina.

Aguado, Empar (2016). «Mujeres en la estiba. El caso de las estibadoras portuarias del puerto de Valencia». Arxius de ciències socials, 35: 123-138.

Collins, Joshua C. (2015). «Characteristics of "Masculinized” Industries: Gay Men as a Provocative Exception to Male Privilege and Gendered Rules». Human Resource Development Review, 14(4): 415-441.

Comas d’Argemir, Dolors (1995). Trabajo, género, cultura: la construcción de desigualdades entre hombres y mujeres. Barcelona: Icaria.

Denissen, Amy M. (2010). «The right tools for the job: Constructing gender meanings and identities in the male-dominated building trades». Human Relations, 63(7): 1.051-1.069.

Kanter, Rosabeth Moss (1977). Men and women of the corporation. Nueva York: Basic Books.

Kimmel, Michael S. (2008). «Homofobia, temor, vergüenza y silencio en la identidad masculina». En: Valdés, Teresa y Olavarría, José (eds.) (2008). Masculinidad/es: poder y crisis. Santiago de Chile: ISIS Internacional.

Lupton, Ben (2000). «Maintaining Masculinity: Men who do "Women's Work" ». British Journal of Management, 11: S33-S48.

Miller, Gloria E. (2004). «Frontier masculinity in the oil industry: The experience of women engineers». Gender, Work and Organization, 11(1): 47-73.

Moreno, Isidoro (1997). «Trabajo, ideologías sobre el trabajo y culturas del trabajo». Revista Andaluza de Relaciones Laborales, 3: 9-28.

OIT (2016). Las mujeres en el trabajo. Tendencias de 2016. Ginebra: OIT.

Palermo, Hernán (2017). La producción de la masculinidad en el trabajo petrolero. Buenos Aires: Biblos.

Palermo, Hernán (2015). «Machos que se la bancan: masculinidad y disciplina fabril en la industria petrolera argentina». Desacatos, 47: 100-115.

Peterson, Helen (2007). «Gendered work ideals in Swedish IT firms: Valued and not valued workers». Gender, Work and Organization, 14(4):333-348.

Pontón Merino, Paloma (2014). «Igualtat de gènere i organitzacions. Una avaluació dels continguts de gènere a la negociació col-lectiva de la industria 
química del camp de Tarragona» [Tesis doctoral]. Universidad Rovira i Virgili, Tarragona, España.

Sтовве, Lineke (2005). «Doing Machismo: Legitimating Speech Acts as a Selection Discourse». Gender, Work and Organization, 12(2)ः 985-1.002.

Téllez, Anastasia (2001). «Trabajo y representaciones ideológicas de género.

Propuesta para un posicionamiento analítico desde la antropología cultural». Gazeta de Antropología, 17: 1-14.

West, Candance y Zimmerman, Don H. (1987). «Doing Gender»+ Gender E Society, 1(2): 125-151.

Wilson, Fiona y Thompson, Paul (2001). «Sexual harassment as an exercise of power». Gender, Work and Organization, 8(1):61-83.

Wright, Tessa (2016). Women's Experience of Workplace Interactions in Male-

Dominated Work: The Intersections of Gender, Sexuality and Occupational Group. Gender, Work and Organization, 23(3): 348-362.

- (2014). "Gender, sexuality and male-dominated work: the intersection of long-hours working and domestic life». Work, Employment E Society, 28(6): 985-1.002.

Ward, Michael RM. (2015). «The Chameleonisation of Masculinityः Jimmy's Multiple Performances of a Working-Class Self». Masculinities and Social Change, 4(3): 215-240.

Zimmer, Lynn (1988). "Tokenism and women in the workplace: the limits of gender-neutral theory». Social problems, 35(1): 64-77.

\section{Recursos electrónicos}

«Hombres empleados en la industria». Banco Mundial. [Consulta: 18 de enero de 2018]. Disponible en: <https;//data.worldbank.org/indicator/SL.IND. EMPL.MA.ZS?year_high_desc=false $>$

«Industria química: empleos bien pagados y muy estables». Diari de Tarragona, 13 de abril de 2017. [Consulta: 18 de abril de 2018]. Disponible en: <https://www.diaridetarragona.com/noticias/Industria-quimica-empleosbien-pagados-y-muy-estables-20170413-0007.html>

«Mujeres empleadas en la industria». Banco Mundial. [Consulta: 12 de febrero de 2018]. Disponible en: <https;//data.worldbank.org/indicator/SL.IND. EMPL.FE.ZS?year_high_desc=false $>$

«Ocupación por sexo y rama de actividad, España 2016». INE [Consulta: 12 de febrero de 2018]. Disponible en: <http://www.ine.es/jaxiT3/Datos. htm?t $=4875$ > 
«Protocol per a la prevenció i abordatge de l'assetjament sexual i per raó de sexe a l'empresa». Generalitat de Catalunya 2015 [Consulta: 23 de abril de 2018]. Disponible en: <http://treball.gencat.cat/web/.content/13_-_consell_relacions_laborals/documents/04___recursos/publicacions/protocol_assetjament/Protocol-per-a-la-prevencio-i-abordatge-de-lassetjament-sexual-iper-rao-de-sexe-a-lempresa_DEF.pdf >

Revista Viu la Química, número 2, 2016. VIUafons. [Consulta: 18 de abril de 2018]. Disponible en: <http://viuafons.cat/portfolio-item/viu-quimica-2/> 